\title{
Quantum-behaved Particle Swarm Optimization Algorithm for Solving Nonlinear Equations
}

\author{
Xiaofeng Zhang \\ College of Information Engineering, Nanchang \\ Hangkong University \\ Nanchang, China \\ xfzhang@163.com
}

\author{
Guifang Sui \\ College of Information Engineering, Nanchang \\ Hangkong University \\ Nanchang, China \\ sgf320428@126.com
}

\begin{abstract}
A quantum-behaved particle swarm optimization algorithm is presented in this paper for solving nonlinear equations. The positions of particle are coded by probability amplitudes of qubits that are updated by quantum rotation gates in this method. The corresponding real number solution at specified interval can be extracted by this algorithm for solving nonlinear equations. Compared to real traditional method, the simulation results show that this algorithm is more accurate and effective.
\end{abstract}

Keywords-Quantum Particle Swarm Optimization, Quantum rotation gates, Nonlinear equations, Function optimization

\section{INTRODUCTION}

Most of engineering problems can be described by nonlinear equations. Therefore, there is a necessity to investigate such numerical methods in order to provide approximate solutions as well as meeting certain precision. Worldwide researchers have been dedicating to developing more effective methods to solve nonlinear equations. However, these methods can only be used by meeting some rigorous conditions, which also have some drawbacks for the case of solving multi-dimensional complex equations. For instance, Newton method having the second order convergence is an important method for solving nonlinear equations, perhaps you can sentence with red color which demands that initial vector is selected in the adjacent solutions. If the initial vector selection is inaccurate, Newton method may be non-convergence and time costing, causing the local extremism.

Particle Swarm Optimization(PSO) is a new global optimized evolutionary algorithm, which is suggested by American psychologist Kennedy and electrical engineer Eberhart who were inspired by the predatory behavior of birds in 1995[1]. However, in practical application, PSO algorithm has some shortcomings, such as the ease to generate premature convergence, long computational time, and low global optimization capability. In order to overcome such drawbacks, a lot of efforts have been made to improve PSO algorithm, but there is premature convergence phenomenon [2]. Quantum-inspired evolutionary algorithm(QEA) based on the concept and theory of quantum computing [3] is a new probability evolutionary algorithm, obtained from observing the quantum state of chromosomes to generate the needed binary solution. However, it is a probabilistic procedure with a great deal of randomness. In order to avoid the observation of the randomness and frequent decoding from binary to decimal, probability amplitude of qubit is directly expressed as solutions of optimization problems in Ref.[4]. Therefore, a Quantum Particle Swarm Optimization(QPSO) algorithm is presented here by incorporating PSO with QEA. This algorithm directly codes position of particle by probability amplitudes of qubits and updates probability amplitude of qubits by quantum rotation gates to realize update of the particle location, search of the optimal position and the variation of qubits by quantum NOT gate, which can increase the diversity of the population and avoid premature convergence. Because each qubit has two probability amplitudes, each particle occupies both locations of the optimization space. Meanwhile, due to the use of double probability amplitude encoding mechanism, the chance for particles to locate their optimal positions is increased significantly. This algorithm has a strong search capability and optimizing efficiency, especially for complex functions optimization and highdimensional optimization problems. In this case, solving nonlinear equations is transformed into the function extreme value optimization problems in this paper, and subsequently quantum-behaved particle swarm optimization algorithm is proposed for solving nonlinear equations. This method improves algorithmic optimization speed, local search ability and global optimization ability.llow.

\section{QUANTUM CALCULATION}

\section{A. Qubits}

A qubit is defined as the smallest unit of information in QEA, which can be described by a pair of numbers $(\alpha, \beta)$ as $[\alpha, \beta]^{T}$. A qubit may be in the $|0\rangle$ state, in the $|1\rangle$ state, or in any linear superposition of the two. The state of a qubit can be represented as $|\phi\rangle=\alpha|0\rangle+\beta 1\rangle$, where $|\alpha|^{2}+|\beta|^{2}=1,|\alpha|^{2}$ gives the probability of the qubit that will be found in the $|0\rangle$ state and $|\beta\rangle$ gives the probability of the qubit that will be found in the $|1\rangle$ state. Therefore, by probability amplitude qubits can be expressed as $|\phi\rangle=[\alpha, \beta]^{T}$. 


\section{B. Quantum Gates}

In quantum calculation, we can achieve some logic functions by carrying out a series of unitary transformation with quantum state. Since the role of the transformation is equivalent to the role of logic gates, remove so quantum gate is quantum device of logical transformation within a certain time interval. Quantum gate is the basis of physics implement quantum computing, and contains the characteristics of quantum computing. There is existing proof [5] that the quantum gates contain universal quantum gates groups, which is similar to the commonality of NAND gate of classic bit. Consequently, the arbitrary quantum gates can be composed of these quantum gates groups. It should be noted that the most basic universal quantum gates group is composed of one phase shift gate and two controlled NOT gate.

First, one phase shift gate is defined as

$$
R(\theta)=\left[\begin{array}{cc}
\cos \theta & -\sin \theta \\
\sin \theta & \cos \theta
\end{array}\right]
$$

the quantum state

$$
|\phi\rangle=\left[\begin{array}{c}
\cos \theta_{0} \\
\sin \theta_{0}
\end{array}\right]
$$

When $R(\theta)$ act on $|\phi\rangle$, then

$$
R(\theta)|\phi\rangle=\left[\begin{array}{c}
\cos \left(\theta_{0}+\theta\right) \\
\sin \left(\theta_{0}+\theta\right)
\end{array}\right]
$$

Obviously $R(\theta)$ plays the role of phase shift to realize the search of optimal location of the particles in solving equations.

The role of quantum NOT gate is to exchange two qubits probability amplitudes, which is defined as

$$
U=\left[\begin{array}{ll}
0 & 1 \\
1 & 0
\end{array}\right]
$$

Then

$$
\begin{aligned}
& U|\phi\rangle=\left[\begin{array}{c}
\sin \theta_{0} \\
\cos \theta_{0}
\end{array}\right]=\left[\begin{array}{c}
\cos \left(\frac{\pi}{2}-\theta_{0}\right) \\
\sin \left(\frac{\pi}{2}-\theta_{0}\right)
\end{array}\right] \\
& =R\left(\frac{\pi}{2}-2 \theta_{0}\right)|\phi\rangle
\end{aligned}
$$

Quantum NOT gate plays a role as a phase rotation in solving equations, which realizes particle position variation to avoid premature convergence. Therefore controlled NOT gate [6] can be constructed as

$$
C(k)=\left[\begin{array}{cc}
\cos \left(\frac{k \pi}{2}-2 \theta_{0}\right) & -\sin \left(\frac{k \pi}{2}-2 \theta_{0}\right) \\
\sin \left(\frac{k \pi}{2}-2 \theta_{0}\right) & \cos \left(\frac{k \pi}{2}-2 \theta_{0}\right)
\end{array}\right]
$$

According to the value of controlled parameter $\mathrm{k}$, its controlled effect can be expressed as

$$
c(k)=\left\{\begin{array}{cc}
{\left[\sin \theta_{0}, \cos \theta_{0}\right]^{T}} & k=1 \\
{\left[\cos \theta_{0}, \sin \theta_{0}\right]^{T}} & k=0 \\
\text { else } & 0<k<1
\end{array}\right.
$$

\section{QUANTUM PARTICLE SWARM AlgORITHM}

\section{A. Initializing Population}

In QPSO, due to the code randomness in population initialization, we code the quantum current position using the probability amplitude of qubits, giving rise to:

$$
\left|P_{i}\right\rangle=\left[\begin{array}{c|c|c|c|}
\cos \theta_{i 1} & \cos \theta_{i 2} & \cdots & \cos \theta_{i n} \\
\sin \theta_{i 1} & \sin \theta_{i 2} & \cdots & \sin \theta_{i n}
\end{array} \mid\right]
$$

where $\theta_{i j}=2 \pi \times r n d$; rnd is the random number between 0 and 1 , where $i=1,2, \ldots, m ; j=1,2, \ldots, n$; $m$ is the population size; $\mathrm{n}$ is the number of spatial dimensions.

\section{B. Solution Space Transformation}

In QPSO, due to the calculation of particle current advantages and disadvantages, we need to transform the solution space. Using linear transformation, remove 'the' every probability amplitude of qubits can be mapped from the unit space into the solution space of optimization problem. However, given that each probability amplitude of particle corresponds to an optimized variable of the solution space, we define the domain of the variable $X_{j}$ of optimization problem and the the j-th qubit of particle $\left|P_{i}\right\rangle$ to be $\left[a_{i}, b_{i}\right]$ the $\mathrm{j}$-th qubit of particleis and $\left[\alpha_{j}^{i}, \beta_{j}^{i}\right]^{T}$, respectively. The corresponding solution space variable is given by:

$$
\begin{aligned}
& {\left[\begin{array}{l}
X_{j 1}^{i} \\
X_{j 2}^{i}
\end{array}\right]=\left[\begin{array}{cc}
\frac{b_{i}-a_{i}}{2} & 0 \\
0 & \frac{b_{i}-a_{i}}{2}
\end{array}\right]\left[\begin{array}{l}
\alpha_{j}^{i} \\
\beta_{j}^{i}
\end{array}\right]+\left[\begin{array}{c}
\frac{b_{i}+a_{i}}{2} \\
\frac{b_{i}+a_{i}}{2}
\end{array}\right]} \\
& \text { Namely } \\
& \left\{\begin{array}{l}
X_{j 1}^{i}=\frac{1}{2}\left[b_{j}\left(1+\alpha_{j}^{i}\right)+a_{j}\left(1-\alpha_{j}^{i}\right)\right] \\
X_{j 2}^{i}=\frac{1}{2}\left[b_{j}\left(1+\beta_{j}^{i}\right)+a_{j}\left(1-\beta_{j}^{i}\right)\right]
\end{array}\right.
\end{aligned}
$$




\section{Updating Particle State}

In QPSO, updates of particles state can be described as the updates of qubit argument angle incremental in each particle and the updates of qubit probability amplitude of quantum rotation gates. Such a strategy is shown as follows:

We let the particles $\left|P_{i}\right\rangle$ search for the optimal location $\left|P_{l}\right\rangle$, and the entire population search for the optimal location $\left|P_{g}\right\rangle$. The update of qubit argument angle incremental in particle $P_{i}$ is

$$
\Delta \theta_{i j}(t+1)=w \Delta \theta_{i j}(t)+c_{1} r_{1}\left(\Delta \theta_{l}\right)+c_{2} r_{2}\left(\Delta \theta_{g}\right)
$$

definition

$$
\Delta \theta_{k}=\left\{\begin{array}{cc}
2 \pi+\theta_{k j}-\theta_{i j} & \theta_{k j}-\theta_{i j}<-\pi \\
\theta_{k j}-\theta_{i j} & -\pi<\theta_{k j}-\theta_{i j}<\pi \\
\theta_{k j}-\theta_{i j}-2 \pi & \theta_{k j}-\theta_{i j}>\pi
\end{array}\right.
$$

where $\mathrm{i}=1,2, \cdots, \mathrm{m} ; \mathrm{j}=1,2, \cdots, \mathrm{n}$; $\mathrm{k}=\mathrm{l}$, g; $\mathrm{w}$ is the inertia factor, $C_{1}, C_{2}$ are constant, $C_{1}$ is own factor and $C_{2}$ is global factor; $r_{1}, r_{2}$ are the random numbers between 0 and 1 , $\Delta \theta_{l}$ is qubit argument angle incremental of the particle that search for the optimal position, $\Delta \theta_{g}$ is qubit argument angle incremental of the entire population that search for the optimal position. The update of qubit probability amplitude of quantum rotation gates in particles $\left|P_{i}\right\rangle$ is

$$
\begin{aligned}
& {\left[\begin{array}{c}
\cos \left(\theta_{i j}(t+1)\right) \\
\sin \left(\theta_{i j}(t+1)\right)
\end{array}\right]=R\left(\Delta \theta_{i j}(t+1)\right)\left[\begin{array}{c}
\cos \left(\theta_{i j}(t)\right) \\
\sin \left(\theta_{i j}(t)\right)
\end{array}\right]} \\
& =\left[\begin{array}{c}
\cos \left(\theta_{i j}(t)+\Delta \theta_{i j}(t+1)\right) \\
\sin \left(\theta_{i j}(t)+\Delta \theta_{i j}(t+1)\right)
\end{array}\right]
\end{aligned}
$$

The angular size and orientation of quantum rotation gates are very important and affect directly the convergence speed and optimization direction. Definition is shown below

$$
\mathrm{A}_{i j}=\left|\begin{array}{cc}
\cos \theta_{l j} & \cos \theta_{g j} \\
\sin \theta_{l j} & \sin \theta_{g j}
\end{array}\right|
$$

the relationship of angle is

$$
\Delta \theta_{i j}=-\operatorname{sgn}\left(\mathrm{A}_{i j}\right) \theta_{0} e^{-t}
$$

where $\mathrm{i}=1,2, \cdots, \mathrm{m} ; \mathrm{j}=1,2, \cdots, \mathrm{n} ; \theta_{0}$ is the initial value, $\mathrm{t}$ is optimization step. The determinating rules of angular orientation[7]: the orientation is $-\operatorname{sgn}\left(\mathrm{A}_{i j}\right)$ when $\mathrm{A}_{i j} \neq 0$, the orientation can be positive and negative when $\mathrm{A}_{i j}=0$. The coding after the particle update is

$$
\left.\left|\tilde{P}_{i}\right\rangle=\left[\begin{array}{c}
\cos \left(\theta_{i 1}(t)+\Delta \theta_{i 1}(t+1)\right) \\
\sin \left(\theta_{i 1}(t)+\Delta \theta_{i 1}(t+1)\right)
\end{array}\right) \cdot \begin{array}{c}
\cos \left(\theta_{i n}(t)+\Delta \theta_{i n}(t+1)\right) \\
\sin \left(\theta_{i n}(t)+\Delta \theta_{i n}(t+1)\right)
\end{array} \mid\right]
$$

\section{Variation Processing}

In QPSO, The quantum NOT gate is adopted to realize particle location variations, to change the probability amplitude of particle location, and to flip particle optimization direction. Meanwhile, by introducing the mutation operator, this method not only avoids the loss of the population diversity in the search process, but also prevents premature convergence and increases the opportunities of particles search for the optimal location. In particle $\left|P_{i}\right\rangle$, the variation of qubit probability amplitude of quantum NOT gate is

$\left[\begin{array}{ll}0 & 1 \\ 1 & 0\end{array}\right]\left[\begin{array}{l}\cos \theta_{i j} \\ \sin \theta_{i j}\end{array}\right]=\left[\begin{array}{l}\sin \theta_{i j} \\ \cos \theta_{i j}\end{array}\right]=\left[\begin{array}{l}\cos \left(\frac{\pi}{2}-\theta_{i j}\right) \\ \sin \left(\frac{\pi}{2}-\theta_{i j}\right)\end{array}\right]$

\section{QPSO FOR SOLVING NONLINEAR EQUATIONS}

\section{A. Problem Description} is

The general mathematical model of nonlinear equations

$$
\left\{\begin{array}{c}
f_{1}\left(x_{1}, x_{2}, \cdots, x_{n}\right)=0 \\
f_{2}\left(x_{1}, x_{2}, \cdots, x_{n}\right)=0 \\
\vdots \vdots \vdots \\
f_{m}\left(x_{1}, x_{2}, \cdots, x_{n}\right)=0
\end{array}\right.
$$

Considering problem: $\min _{x \in R^{n}}\|f(x)\|_{p}$, the $\|\bullet\|_{p}$ is p-norm of vector space, and the $\min _{x \in R^{n}}\|f(x)\|_{p}$ can be taken as the minimum value 0 if and only if $f_{i}\left(x_{1}, x_{2} \cdots x_{n}\right)=0$. So the problem of solving arbitrary multivariate nonlinear equations is transformed into solving multivariate nonlinear minimum value. The solution of (the) minimal value is 0 or close to 0 , which is the solution of the equation $f_{i}\left(x_{1}, x_{2} \cdots x_{n}\right)=0$. We suppose that $\mathrm{p}=1$, then

$$
\min _{x \in R^{n}}\|f(x)\|=\min _{x \in R^{n}}\left(\sum_{i=1}^{m}\left|f_{i}\left(x_{1}, x_{2}, \cdots x_{n}\right)\right|\right)
$$

\section{B. Numerical Experiments}

In order to examine the performance of quantum particle swarm algorithm, the numerical simulation is performed using the examples which is given in Ref. [8]. Simulation parameter parameters are given as follows: population size is 50; quantum bits is 2; limited algebra is 500 ; inertia factor is 0.5 ; autogenous factor is 2; global factor is 2; mutation probability is 0.05 and the nonlinear equations of simulation is

$$
\left\{\begin{array}{c}
e^{4 \sin x_{1}}+e^{2 \cos x_{2}}=2 \\
e^{x_{1}}+\frac{1}{2} e^{x_{2}}=1
\end{array}\right.
$$




\begin{tabular}{|c|c|c|c|c|}
\hline $\begin{array}{c}\text { Literatur } \\
\text { e [8] }\end{array}$ & non & -0.131789 & 770 & Convergence \\
& & 994367941 & & \\
& & -1.39856 & & \\
\hline Fsolve & $(-3,-3)$ & -0.13178946969 & 22 & Convergence \\
function & & 994380944 & & (concern \\
& & -1.39856 & & initial point) \\
& & 980442676 & & \\
\hline Newton & $(-0.1,-0.5)$ & -0.131789 & 5 & convergence \\
method & & 991803407 & & (concern \\
& & -1.39856 & & initial point) \\
& & 984393425 & & Convergence \\
& & -0.131789 & 371 & (operation10t \\
QPSO & non & 993092176 & & convergence \\
& & -1.39856 & & 8times) \\
\hline
\end{tabular}

\section{Results Analysis}

Experimental results show that the traditional methods require the rigorous initial value condition for solving nonlinear equations. If the initial value choice is inaccurate, it will affect function convergence to produce optimal solutions of target function. Although Ref.[8] has no initial value, it limits the search interval of the optimal value. However, since QPSO algorithm does not limit the search interval of optimal solutions, and it is the global search in range of all real numbers field, their approximate solutions are more accurate. Therefore, QPSQ can be regarded as a more effective and accurate method for solving nonlinear equations compared to the traditional methods.

\section{Conclusions}

A quantum particle swarm optimization algorithm is applied to solve nonlinear equations. The capability of such an algorithm to generate faster convergence speed, better objective function and more accurate solution precision than the traditional methods is demonstrated. Particle swarm algorithm as a kind of more effective evolution algorithm is proposed for solving nonlinear equations.

\section{ACKNOWLEDGMENT}

We acknowledge the support from the Natural Science Foundation of China (No.60973048), the Natural Foundation of Jiangxi Province of China (No.2009GZS0084), the Scientific Research Projects of Education Department of Jiangxi Province of China (No.GJJ12413), the Innovation Foundation of Postgraduate of Nanchang Hangkong University (No.YC2011-S097, No.YC2011038, No.YC2011003).

\section{REFERENCES}

[1] Kennedy J, Eberhart R C. Particle swarms optimization. Proc.IEEE International Conference on Neural Networks. USA:IEEE press, 4 (1995), 1942-1948.

[2] Chen B R, Feng X T. Particle swarm optimization with contracted ranges of both search space and velocity. Journal of Northeastern University(Natural Science), 26:5 (2005), 488-491.

[3] Hyun K, Kim J H. Quantum-inspired evolutionary algorithm for a class of combinational optimization. IEEE Transactions on Evolutionary Computing, 6:6 (2002), 580-593.

[4] Li Shi-yong, Li Pan-chi. Quantum Particle swarms algorithm for continuous space optimization. Chinese Journal of Quantum Electronics, 24:5 (2007), 569-574.

[5] Barenco A, et al. Elementary gates for quantum computation. Phys Rev A , 52 (1995), 3457 - 3461.

[6] Xie Guang-jun, Zhou Dian, et al. A Neural Network Model Based on Quantum Gates Cell and Its Applications. Systems EngineeringTheory \& Practice, 5 (2005), 113-117.

[7] Li Shi-yong, Li Pan-chi. Quantum genetic algorithm based on real ecoding and gradient information of objective function. Journal of Harbin Institute of Technology, 38:8 (2006), 1216-1219.

[8] Li Jian-wen, Zhang Cheng-xian, Li Qi et al. Minimum search and sum of squares transformation for solving multivariable nonlinear equations. Journal of Shanxi University of Science and Technology, 28:3 (2010), 143-147. 